\title{
Fast Multi-Objective Optimization of Multi-Parameter Antenna Structures Based on Improved MOEA/D with Surrogate-Assisted Model
}

\author{
Jian Dong ${ }^{1 *}$, Qianqian $\mathrm{Li}^{1}$, Lianwen Deng ${ }^{2}$
}

Abstract-For multi-objective design of multi-parameter antenna structures, optimization efficiency and computational cost are two major concerns. In this paper, an improved multi-objective evolutionary algorithm based on decomposition (MOEA/D) is proposed to improve global optimization capability by diversity detection operation and mixed population update operation. Further, in order to reduce the computational cost, a hybrid optimization strategy integrating a dynamically updatable surrogate-assisted model into the improved MOEA/D is proposed. The numerical results of test functions show that our algorithm outperforms original MOEA/D, modified MOEA/D (M-MOEA/D), and nondominated sorting genetic algorithm II (NGSA-II) in terms of diversity. Experimental validation of Pareto-optimal planar miniaturized multiband antenna designs is also provided, showing excellent convergence and considerable computational savings compared to those previously published approaches.

Keywords: multi-objective optimization, antenna design, MOEA/D, surrogate model

\section{INTRODUCTION}

Contemporary antenna usually requires simultaneous handing of multiple objectives. In order to accurately evaluate the antenna response, a high-fidelity electromagnetic (EM)

\footnotetext{
* Corresponding author: Jian Dong (dongjian@ @ csu.edu.cn)

1 School of Information Science and Engineering, Central South University, Changsha 410083, China

2 School of Physics and Electronics, Central South University, Changsha 410083, China
} 
simulation is necessary. However, accurate EM analysis is computationally expensive due to the need of repetitive parameter sweeps, which poses a large challenge for multi-objective antenna design [1], [2].

Automated multi-parameter antenna optimization using evolutionary algorithms (EAs) provides a new way for antenna designs because it does not need a prior preference regarding design objectives (which is usually either not obtainable or not desirable). EAs have been successfully applied to multi-objective optimization problems (MOP) related to antenna and array designs, such as genetic algorithm (GA) [3]-[5], particle swarm optimization (PSO) [6], [7], and nondominated sorting genetic algorithm (NSGA) [8]. In these schemes, a MOP is either converted into a single objective optimization problem (SOP) using some composition approaches [3]-[7], or treated as a whole [8]. In these implementations, a weight vector responses to one search direction in design space so that computational cost could be unendurable due to low searching efficiency. In [9], a multi-objective evolutionary algorithm based on decomposition (MOEA/D) was proposed for solving MOPs. In this algorithm, a MOP is explicitly decomposed into a number of scalar subproblems which can be optimized simultaneously. By using the information of solutions of neighboring subproblems, simultaneous optimization of these decomposed subproblems results in a considerable reduced computational cost. As one of the most popular multi-objective optimization techniques, MOEA/D has been successfully applied to array synthesis [10], [11]. However, different from array synthesis, the direct utilization of MOEA/D in the multi-parameter antenna designs may be still difficult if high-fidelity EM simulations are involved. Fortunately, the surrogated-based optimization techniques developed recently by S. Koziel et 
al. [1], [2], [12]-[15] have proved to be computationally efficient than the traditional EM-driven approaches.

In this work, aiming at improving optimization efficiency and computational cost, we propose an improved MOEA/D algorithm expedited with surrogate-assisted model for fast multi-objective design of multi-parameter antenna structures. In our improved MOEA/D, a diversity detection operator is adopted to judge the level of population diversity and then a mixed population update operation is performed to increase population diversity while preserving beneficial individuals. The performance of our improved MOEA/D is validated by several test functions. Moreover, in order to greatly reduce the computational cost of antenna design process, a hybrid optimization strategy integrating a dynamically updatable surrogate model into the improved MOEA/D is proposed. This strategy allows us to obtain a set of Pareto-optimal designs at a very low computation cost. The results of a miniaturized multiband planar antenna design are also presented, showing the superiority of our approach in terms of computational cost and solution diversity over other reported approaches.

\section{PROBLEM FORMULATION}

In this section, we briefly describe the formulation of the multi-objective antenna design problem. Generally, the multi-objective design of multi-parameter antenna structures can be stated as a MOP

$$
\left\{\begin{array}{l}
\min F(\boldsymbol{x})=\left(f_{1}(\boldsymbol{x}), f_{2}(\boldsymbol{x}), \ldots, f_{m}(\boldsymbol{x})\right)^{T} \\
\text { s.t. } \boldsymbol{x} \in \boldsymbol{X}
\end{array}\right.
$$

where $f_{k}(\boldsymbol{x}), k=1,2, \ldots, m$ is the $k$ th design objective. A typical objective is to ensure $\left|S_{11}\right|<-10 \mathrm{~dB}$ over a certain frequency band of interest, and to minimize the antenna reflection over that band. Also, there might be some other objectives with respect to antenna size, gain, 
efficiency, and so on. $\boldsymbol{X}$ is a design space, and $\boldsymbol{x}=\left(x_{1}, \ldots, x_{n}\right)$ is a vector of $n$ design variables defining a particular antenna structure. In multi-objective antenna design, the target is to obtain the Pareto front (PF) [1], [2], i.e., multiple designs representing the trade-off between various characteristic of the antenna under consideration.

For multi-objective optimization, any two designs $\boldsymbol{x}$ and $\boldsymbol{y}$ for which $f_{k}(\boldsymbol{x})<f_{k}(\boldsymbol{y})$ and $f_{l}(\boldsymbol{x})<f_{l}(\boldsymbol{y})$ for at least one pair $k \neq l$, are not commensurable, i.e., none is better than the other in the multi-objective sense [1], [2]. We define the Pareto dominance relation $\prec$

saying that for the two designs $\boldsymbol{x}$ and $\boldsymbol{y}$, we have $\boldsymbol{x} \prec \boldsymbol{y}(\boldsymbol{x}$ dominates $\boldsymbol{y})$ if $f_{k}(\boldsymbol{x}) \leq f_{k}(\boldsymbol{y})$ for all $k=1,2, \ldots, m$ and $f_{k}(\boldsymbol{x})<f_{k}(\boldsymbol{y})$ for at least one $k$ [1], [2], [16]. The multi-objective optimization aims to find a representation of a Pareto front $\boldsymbol{X}_{p}$ (viz. Pareto-optimal set) of the design space $\boldsymbol{X}$, such that for any $\boldsymbol{x} \in X_{p}$, there is no $\boldsymbol{y} \in X$ for which $\boldsymbol{y} \prec \boldsymbol{x}$ [1], [2], [16].

\section{DESIGN METHODOLOGY}

\subsection{Improved MOEA/D}

MOEA/D is one of the most popular optimization algorithms in solving MOPs. In MOEA/D implementation, it is necessay to decompose the MOP under consideration [9]. Let $\lambda^{1}, \ldots, \lambda^{N}$ be a set of $N$ evenly distributed weight vectors and $z^{*}$ be the reference point. The problem of the PF approximation in (1) can be decomposed into $N$ scalar optimization subproblems by using several decomposition approaches (e.g., the Tchebycheff approach) and the objective function of the $j$ th subproblem is

$$
g^{t e}\left(x \mid \lambda{ }^{j} z^{*} \neq_{1 \leq i \leq M} \max ^{j} f_{i} *-\left(z^{*}\right\}\right.
$$

where $\lambda^{j}=\left(\lambda_{1}^{j}, \ldots, \lambda_{M}^{j}\right)$. MOEA/D features excellent optimization efficiency by minimizing all 
objective functions simultaneously in a single run.

In MOEA/D, the neighborhood size (viz. $\mathrm{T}$ in [9]) plays a key role in algorithm performance. A smaller $\mathrm{T}$ leads to a good local optimization capability and hence fast convergence. Considering the heavy burden of antenna simulations, a smaller value of $\mathrm{T}$ is usually preferred in multi-parameter antenna design. However, a smaller $\mathrm{T}$ may result in degraded population diversity and tend to prematurity. Accordingly, it is necessary to develop a scheme to improve diversity for a small value of $\mathrm{T}$. In our proposed algorithm, two improvements are introduced: a) a diversity detection operator is designed to judge the level of population diversity; b) population update is performed partially by random disturbance strategy and partially by a nondominated sorting strategy (NDS) in order to improve the diversity while keeping promising individuals. A detailed description of the improvements is given as follows:

\section{Operation a): Diversity Detection}

Variance can measure how far a set of data are spread out in probability theory and statistics. So, we introduce this concept to measure the degree of aggregation of population. First, we define the fitness variance of population, $\sigma^{2}$, as follows,

$$
\sigma^{2}=\frac{1}{M} \frac{1}{N} \sum_{i=1}^{M} \sum_{j=1}^{N}\left(\frac{f_{i j}-f_{i a_{v}}}{f}\right)^{2}
$$

where $f=\max \left\{1, \max \left|f_{i j}-f_{\text {iavg }}\right|\right\},(i \in[1, M], j \in[1, N]), M$ and $N$ are the number of objectives and population size, respectively; $f_{i j}$ and $f_{\text {iavg }}$ are the fitness of the $j$ th individual and the mean fitness of population for the $i$ th objective, respectively. The lower the value of $\sigma^{2}$ is, the more similarities there are among the individuals; and vice versa.

Then by comparing the value of $\sigma^{2}$ with a preset threshold, we can judge whether 
premature occurs or not. We define the threshold function, $H(t)$, as follows

$$
H(t)=H_{\mathrm{max}}-\frac{\left(H_{\mathrm{max}}-H_{\mathrm{m}}\right)_{\mathrm{m}} t}{\text { MaxIter }}
$$

where $H_{\max }$ and $H_{\min }$ are the maximum and minimum thresholds, respectively; $t$ is the iteration index and MaxIter is the number of total iteration. If the value of $\sigma^{2}$ is smaller than the value of $H(t)$, local optimum will occur. Considering that the population diversity decreases with the number of iteration, a time-variant decreasing threshold function is designed. Specifically, a larger threshold is set at the initial stage of evolution to reduce the possibilities of falling into local minima; while with the increase of the iteration number, a gradually decreased threshold is set to track the state of population accurately.

\section{Operation b): Mixed Population Update}

Let $P$ denote the current population and $P_{a}$ denote an alternative population which is used for the storage of promising individuals. When local minima occurs, a sub-population $P_{s}$ consisting of $N_{r}$ individuals is first selected from the current population randomly

$$
N_{r}=\operatorname{rou}(q d \times q)
$$

where $q$ is an alterable re-selection factor, round is a function that rounds to the nearest integer. The duplicate version of sub-population $P_{s}$ is stored in the alternative population $P_{a}$ to avoid missing promising individuals in the next operation of random disturbance on $P_{s}$.

Then, a random disturbance operator is performed on each individual of the sub-population $P_{s}$

$$
\boldsymbol{x}_{k}^{\prime}=\boldsymbol{x}_{k} \times\left(1+\frac{\lambda}{2}\right)
$$

where $\boldsymbol{x}_{k}$ and $\boldsymbol{x}_{k}^{\prime}$ are the previous and updated version of a selected individual $k$ $\left(k=1,2, \ldots, N_{r}\right)$, respectively; $\lambda$ is a random variable with the standard normal distribution in $[0$, 
1]. For the remaining $N-N_{r}$ individuals in $P$, they will be updated together with the alternative population $P_{a}$ by a nondominated sorting strategy (NDS) [17]. Then, the updated population can be expressed as

$$
P=P_{s}+N D\left(\$ P_{s}\right) P U_{a}
$$

It can be seen from (7) that both selected individuals with random disturbance and those promising individuals with NDS are applied to the next evolution to increase the population diversity and meanwhile avoid the loss of promising individuals. The main procedures of the improved MOEA/D are summarized as follows:

Step 1) Initialize a population $P$ with $N$ individuals and decompose weight vectors;

Step 2) Update the population:

Step 2.1) Update the population with genetic operator;

Step 2.2) Detect the population diversity according to population fitness variance and predefined threshold function;

Step 2.3) If premature occurs, update the population according to (5)-(7);

Step 3) Stop the optimization until the maximum iteration number is reached.

\subsection{Improved MOEA/D with Dynamically Updatable Surrogate-Assisted Model}

As mentioned previously, direct use of the high-fidelity EM simulations is not advisable in multi-objective antenna optimization because of heavy computation burden. Therefore, a surrogate model replacing the time-consuming process of EM simulations is highly appreciated. Here a kriging-based interpolation model is introduced, which can predict unknown points by utilizing the known points. The detailed description of the kriging 
interpolation model can be found in [14], [15], [18].

Aiming at fast multi-objective antenna optimization for multi-parameter antenna structures, we establish an optimization framework integrating our improved MOEA/D with a dynamically updatable kriging interpolation model. First, Latin Hypercube Sampling (LHS) [19], [20] is introduced to sample design space. Next, we acquire the high fidelity response set $\boldsymbol{R}_{c d}$ of sampling points by using EM simulation software HFSS and construct an initial kriging interpolation model $\boldsymbol{R}_{s}$ based on $\boldsymbol{R}_{c d}$ [12]-[15]. Compared to randomized sampling, LHS provides surrogate-model with higher prediction accuracy because of more uniformly distributed sampling points in the design space. Then, in the optimization process of improved MOEA/D, the fitness value of each individual is evaluated by the interpolation model $\boldsymbol{R}_{s}$ instead of by the direct EM simulation model for highly reducing the computation cost. Finally, a set of Pareto-optimal designs with respect to the objectives of interest are found.

The prediction accuracy of surrogate-assisted model is a key issue in terms of antenna optimization. To improve its prediction accuracy, it is necessary to update the surrogate model by adding new sampling points as well as their responses into $\boldsymbol{R}_{s}$ during the optimization process. The selection of new sampling points is critical for antenna optimization efficiency. First, a proper distance should be kept between the current samples in $\boldsymbol{R}_{s}$ and newly added samples for saving the computation cost. Second, the newly added samples should have good fitness in terms of the optimization objectives for speeding up the convergence. Based on these concerns, a selection strategy is presented as follows:

1) Select $r$ excellent individuals as the candidate new samples from the current 
population exploiting a NDS strategy;

2) For each selected individual $\boldsymbol{x}_{i}(i=1,2, \ldots, r)$ with $d$-dimension ( $d$ is the number of optimization variables), we find a point $\boldsymbol{z}_{s}$ in the current $\boldsymbol{R}_{s}$ which is closest to this individual in the sense of the first dimension, i.e.,

$$
\left|\boldsymbol{x}_{i, 1}-\boldsymbol{z}_{s, 1}\right|=\min _{z_{j} \in \boldsymbol{R}_{s}}\left|\boldsymbol{x}_{i, 1}-\boldsymbol{z}_{j, 1}\right| j=1,2, \ldots, p
$$

where $p$ is the number of points in the current $\boldsymbol{R}_{s}$.

3) If $\boldsymbol{x}_{i}$ satisfies the following condition (9), $\boldsymbol{x}_{i}$ will be added into $\boldsymbol{R}_{s}$. Otherwise, it will be discarded.

$$
\exists\left|x_{i, k}-z_{s, k}\right|>\max _{z_{j} \in R_{s}}\left|z_{s, k}-z_{j, k}\right| j=1,2, \cdots, p ; k=2, \ldots, d
$$

Therefore, the entire design can be summarized as follows:

1. Predefine the design space $\boldsymbol{X}$;

2. Sample design space using LHS and acquire the response set $\boldsymbol{R}_{c d}$;

3. Construct an initial kriging-based surrogate model $\boldsymbol{R}_{s}$;

4. Optimize the population by improved MOEA/D with a dynamically updatable kriging-assisted model;

5. If termination condition is not satisfied then go to 4; else end optimization.

The flowchart of the improved MOEA/D with a dynamically updatable surrogate-assisted model is shown in Fig. 1. It is expected that our approach will greatly reduce the computational cost of antenna optimization process and meanwhile maintain population diversity well.

\section{NUMERICAL VERIFICATION AND SIMULATION ANALYSIS}

In this section, we conduct verification of the proposed method in section 3 . First, the 


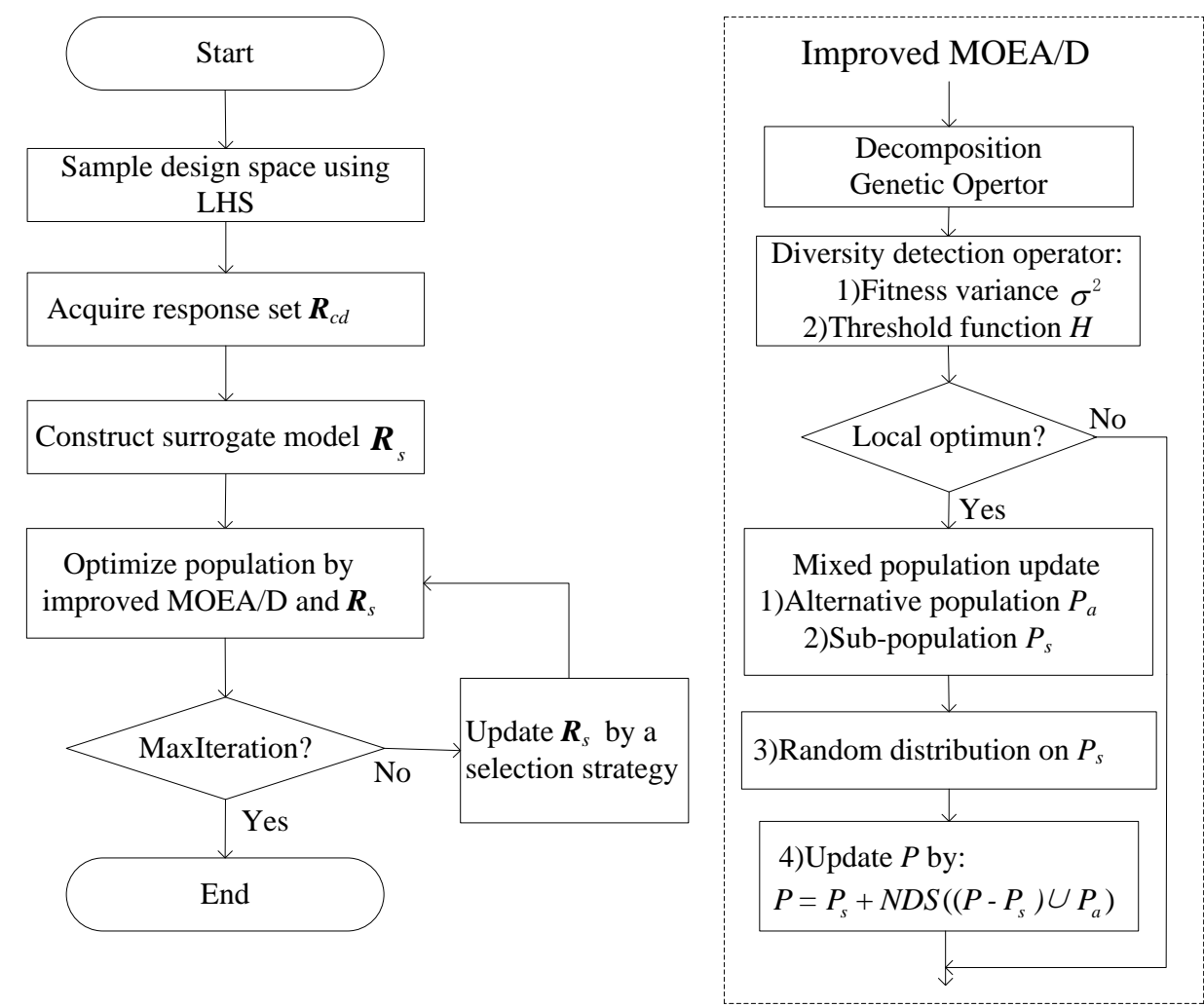

Fig. 1. Flowchart of the improved MOEA/D with a dynamically updatable surrogate-assisted model

performance of our improved MOEA/D is tested using five widely-used 2-objective ZDT test instances [21] and three 3-objective DTLZ test instances [22]. We compare the test results with original MOEA/D [9], modified MOEA/D (M-MOEA/D) [23], and nondominated sorting genetic algorithm II (NGSA-II) [17] to show the effectiveness of our improved MOEA/D. Further, our method is illustrated using a miniaturized multiband antenna design example with 21 parameters. In particular, we emphasize the computational advantages of our technique compared to the conventional antenna design approaches based on other evolutionary algorithms or EM simulation.

\subsection{Test Functions}

Here, we use ZDT1 ZDT4, ZDT6 and DTLZ1 DTLZ2 as the test functions, all these test instances are minimization of the objectives. The population size $N$ is set to be 200 for all 
2-objective test instances and 300 for all three 3-objective test instances. The dimensions of design variables are 30 and 10, respectively. The initial population is generated randomly in the tests. All the algorithms stop after 200 generations. Besides, re-selection factor $q$ in our improved MOEA/D is limited to the interval $(0,0.4)$.

To make comparison among different algorithms, the distance from representatives in the PF (D-metric) [24] is used as figure of merit. Let $P^{*}$ be a set of uniformly distributed points in the objective space along the $\mathrm{PF}$ and $A$ be an approximation to the $\mathrm{PF}$, then the average distance from $P^{*}$ to $A$ is defined as

$$
D\left(A, P^{*} \neq \frac{\sum_{v \in P^{*}} d(v, A}{\left|P^{*}\right|}\right.
$$

where $d(v, A)$ is the minimum Euclidean distance between $v$ and the points in $A$. The lower the D-metric values are, the better both diversity and convergence are. Table I presents the mean and standard deviation of D-metric values of the final solutions obtained by each algorithm for each test instance. In this table, all the reported values have been obtained by averaging over 20 trials. Number in the parentheses in the instance column indicates the number of objectives considered in the corresponding test. For example, ZDT1(2) means ZDT1 test instance with two objectives. Original MOEA/D, M-MOEA/D, and our improved MOEA/D find D-metric values of the solutions using the Tchebycheff approach.

TABLE I Mean and standard deviation of D-metric values of the final populations for different tests

\begin{tabular}{ccccccccc}
\hline \multirow{2}{*}{ Instance } & \multicolumn{2}{c}{ Improved MOEA/D } & \multicolumn{2}{c}{ M-MOEA/D } & \multicolumn{2}{c}{ Original MOEA/D } & \multicolumn{2}{c}{ NSGA-II } \\
\cline { 2 - 8 } & Mean & Std. & Mean & Std. & Mean & Std. & Mean & Std. \\
\hline ZDT1(2) & 0.0042 & 0.0012 & 0.0102 & 0.0042 & 0.0055 & 0.0039 & 0.0027 & 0.0005 \\
ZDT2(2) & 0.0055 & 0.0013 & 0.0118 & 0.0030 & 0.0065 & 0.0024 & 0.0032 & 0.0005 \\
ZDT3(2) & 0.0049 & 0.0032 & 0.0189 & 0.0097 & 0.0124 & 0.0089 & 0.0057 & 0.0048 \\
ZDT4(2) & 0.0076 & 0.0026 & 0.0113 & 0.0047 & 0.0089 & 0.0032 & 0.0157 & 0.0192 \\
ZDT6(2) & 0.0062 & 0.0008 & 0.0062 & 0.0014 & 0.0056 & 0.0012 & 0.0132 & 0.0029 \\
DTLZ1(3) & 0.0302 & 0.0016 & 0.0350 & 0.0029 & 0.0347 & 0.0007 & 0.0625 & 0.0913
\end{tabular}




\begin{tabular}{lllllllll} 
DTLZ2(3) & 0.0298 & 0.0015 & 0.0258 & 0.0024 & 0.0386 & 0.0005 & 0.0507 & 0.0024 \\
DTLZ3(3) & 0.0085 & 0.0012 & 0.0042 & 0.0013 & 0.0102 & 0.0019 & 0.0144 & 0.0098 \\
\hline \hline
\end{tabular}

From Table I, we have the observation that improved MOEA/D has lower D-metric values than M-MOEA/D for all instances except for DTLZ2(3) and DTLZ3(3), and has lower D-metric values than original MOEA/D for all instances except for ZDT6(2). NSGA-II has lower values than improved MOEA/D only for ZDT1(2) and ZDT2(2). Therefore, it can be concluded that improved MOEA/D works better in terms of population diversity than original MOEA/D and NSGA-II in solving 2-objective and 3-objective instances, and works better for 2-objective instances and slightly worse for several 3-objective instances than M-MOEA/D. Also, it should be mentioned that improved MOEA/D is more stable than M-MOEA/D for all instances, and is more stable than original MOEA/D for all instances except for DTLZ1(3) and DTLZ2(3), and is more stable than NSGA-II except for ZDT1(2) and ZDT2(2).

Fig. 2 presents the evolution curves of the average D-metric value for each algorithm at each test instance. These results indicate that improved MOEA/D converges much faster than M-MOEA/D except for DTLZ2(3) and DTLZ3(3). Also, improved MOEA/D converges faster than original MOEA/D except for ZDT6(2), and converges faster than NSGA-II except for ZDT1(2) and ZDT2(2). Table II presents the average CPU time per iteration used by each algorithm at each test instance. It can be concluded that improved MOEA/D has better diversity than original MOEA/D at a negligible added computation cost, and has better diversity and much smaller computational cost than NSGA-II, especially for the 3-objective test instances. Also, compared to M-MOEA/D, improved MOEA/D has much better diversity and less computation cost for all 2-objective instances, and has similar diversity and computation cost for 3-objective instances. 

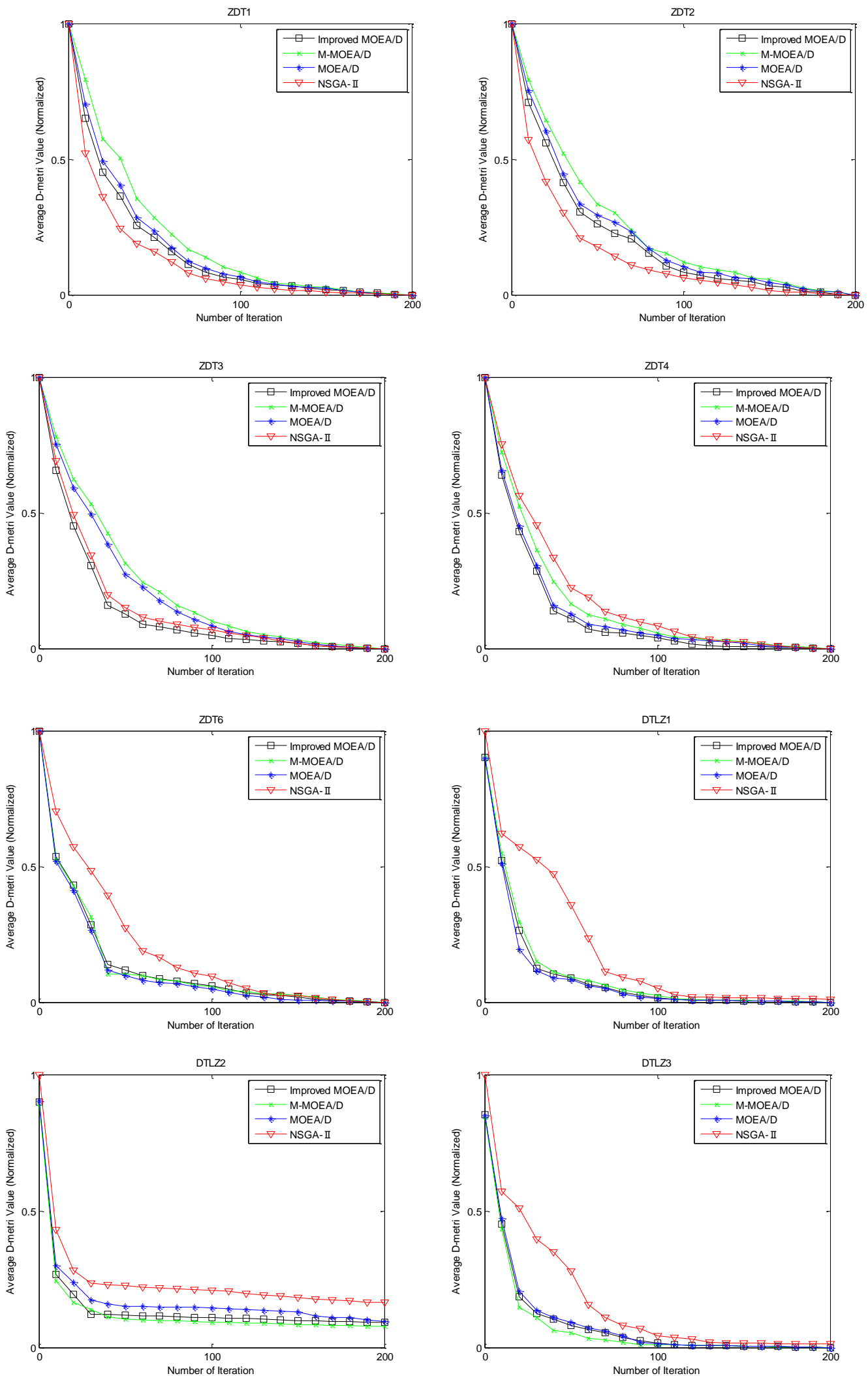

Fig. 2. The evolution of average D-metric value in improved MOEA/D, M-MOEA/D, original MOEA/D and NSGA-II for each test instance 
TABLE II Average CPU time per iteration (in seconds) used by each algorithm for different tests

\begin{tabular}{ccccc}
\hline \hline Instance & Improved MOEA/D & M-MOEA/D & Original MOEA/D & NSGA-II \\
\hline ZDT1(2) & 0.65 & 0.69 & 0.61 & 1.18 \\
ZDT2(2) & 0.60 & 0.65 & 0.58 & 1.15 \\
ZDT3(2) & 0.66 & 0.72 & 0.62 & 1.17 \\
ZDT4(2) & 0.42 & 0.48 & 0.41 & 0.92 \\
ZDT6(2) & 0.42 & 0.44 & 0.40 & 0.91 \\
DTLZ1(3) & 1.25 & 1.25 & 1.23 & 10.29 \\
DTLZ2(3) & 1.13 & 1.14 & 1.11 & 8.42 \\
DTLZ2(3) & 1.31 & 1.31 & 1.29 & 12.37 \\
\hline \hline
\end{tabular}

4.2 Miniaturized multiband antenna design

Our method is illustrated using a miniaturized multiband antenna design example. Miniaturized multiband antennas are highly desirable in modern wireless communications because they can operate at different frequency bands and allow integrating different communication standards into a single small portable device. Fig. 3 shows the geometry of our previous designed multiband antenna [25]. This antenna is composed of a dielectric substrate, a T-shaped microstrip patch with a circle slot, and a comb-shaped ground on the back of the substrate. By employing these structures, the antenna can yield three different resonances to cover the desired bands. The antenna is implemented on a FR-4 substrate of thickness 1.6mm, permittivity 4.4, and loss tangent 0.02 . Design variables are

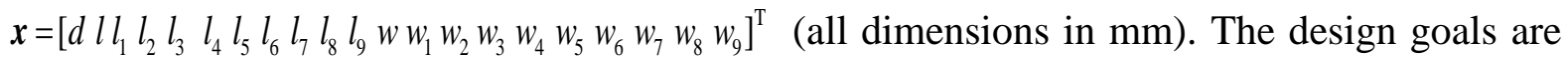
(i) the values of $S_{11}$ are lower than $-10 \mathrm{~dB}$ for three bands (objective $F_{1}$ ), covering the entire WLAN2.4/5.2/5.8GHz, WiMAX2.5/3.5/5.5GHz, and X-band SATcom applications (7.9 8.4GHz); and (ii) reduction of a structure footprint (objective $F_{2}$ ) for meeting the need of antenna minimization in mobile devices. Compared to antenna designs in [23], much more geometry parameters are to be optimized, and structure footprint minimization is also 
considered besides realizing multi-band properties.

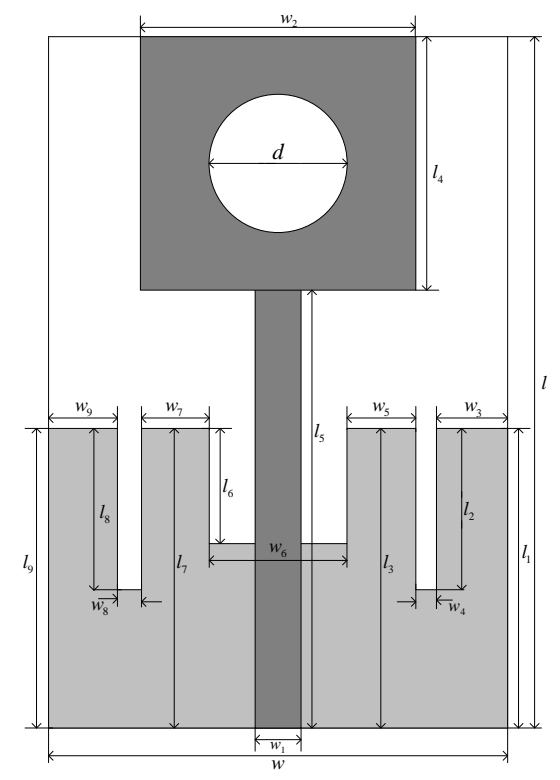

Fig. 3. Geometry of the planar multiband antenna

The objective function of $F_{1}$ can be specified as

$$
\begin{gathered}
Q\left(f_{i}\right)= \begin{cases}\left|S_{11}\left(f_{i}\right)\right| & \left|S_{11}\left(f_{i}\right)\right|>-10 \\
-10 & \left|S_{11}\left(f_{i}\right)\right| \leq-10\end{cases} \\
F_{1}=\frac{1}{n} \sum_{i=1}^{n} Q\left(f_{i}\right)
\end{gathered}
$$

where $f_{i}$ is the frequency of the $i$ th sampling points within the given frequency ranges; $S_{11}\left(f_{i}\right)$ is the reflection coefficient of sampling point $f_{i} ; n$ is the number of sampling points. The objective function of $F_{2}$ can be defined as

$$
F_{2}=w \times l
$$

The initial optimization ranges of design parameters are shown in Table III. A kriging interpolation model $\boldsymbol{R}_{\mathrm{S}}$ has been constructed in the design space using the samples allocated with LHS. The model $\boldsymbol{R}_{\mathrm{s}}$ is updated dynamically in the optimization process and the Pareto optimal set has been subsequently found using improved MOEA/D with surrogate-assisted model. The obtained representations of the Pareto set are shown in Fig. 4. 
TABLE III Initial ranges of design parameters (units: mm)

\begin{tabular}{cccccccc}
\hline \hline Parameter & $d$ & $l$ & $l_{1}$ & $l_{2}$ & $l_{3}$ & $l_{4}$ & $l_{5}$ \\
Range & {$[7,10]$} & {$[26,34]$} & {$[11,14]$} & {$[8,10]$} & {$[11,14]$} & {$[10,14]$} & {$[16,20]$} \\
Parameter & $l_{6}$ & $l_{7}$ & $l_{8}$ & $l_{9}$ & $w$ & $w_{1}$ & $w_{2}$ \\
Range & {$[6,8]$} & {$[11,14]$} & {$[8,10]$} & {$[11,14]$} & {$[17,23]$} & {$[2,4]$} & {$[11,13]$} \\
Parameter & $w_{3}$ & $w_{4}$ & $w_{5}$ & $w_{6}$ & $w_{7}$ & $w_{8}$ & $w_{9}$ \\
Range & {$[2,4]$} & {$[0.5,1.5]$} & {$[2,4]$} & {$[4,7]$} & {$[2,4]$} & {$[0.5,1.5]$} & {$[2,4]$} \\
\hline \hline
\end{tabular}

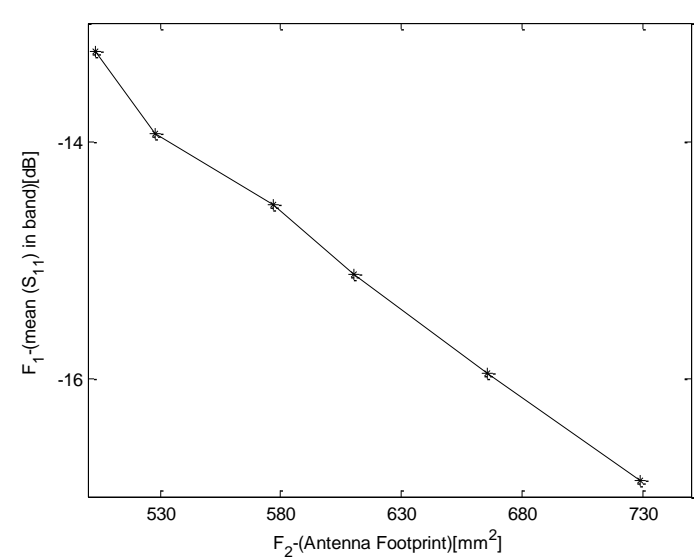

Fig. 4. The obtained representations of the Pareto set for the planar multiband antenna

The obtained results indicate that the design objectives considered for this problem are indeed conflicting. In particular, the antenna design with the lowest in-band reflection of $-16.87 \mathrm{~dB}$ simultaneously exhibits the largest footprint of $729.17 \mathrm{~mm}^{2}$. Also, the design with the highest acceptable in-band reflection level of $-13.24 \mathrm{~dB}$ is characterized by the smallest occupied area $\left(503.30 \mathrm{~mm}^{2}\right)$. Note that the reflection and footprint vary by $3.63 \mathrm{~dB}$ and $30.98 \%$ along the Pareto-front, respectively. The detailed data on the optimized designs is gathered in Table IV, and their reflection responses are shown in Fig. 5. All these designs provide flexible choices for practical antenna engineering. 
TABLE IV Planar multiband antenna: selected Pareto-optimal designs

\begin{tabular}{|c|c|c|c|c|c|c|}
\hline Design & $\boldsymbol{x}^{(1)}$ & $\boldsymbol{x}^{(2)}$ & $\boldsymbol{x}^{(3)}$ & $x^{(4)}$ & $\boldsymbol{x}^{(5)}$ & $\boldsymbol{x}^{(6)}$ \\
\hline$F_{1}[d B]$ & -16.87 & -15.96 & -15.12 & -14.54 & -13.93 & -13.24 \\
\hline$F_{2}\left[m^{2}\right]$ & 729.17 & 665.56 & 610.07 & 576.91 & 527.81 & 503.30 \\
\hline$d$ & 9.21 & 8.98 & 8.80 & 8.12 & 8.45 & 8.04 \\
\hline$l$ & 32.25 & 30.87 & 29.92 & 29.57 & 28.18 & 27.73 \\
\hline$l_{1}$ & 13.76 & 12.85 & 13.21 & 13.12 & 12.76 & 12.53 \\
\hline$l_{2}$ & 9.38 & 8.97 & 9.25 & 9.13 & 8.93 & 8.66 \\
\hline$l_{3}$ & 13.61 & 12.96 & 12.98 & 13.05 & 12.89 & 12.49 \\
\hline$l_{4}$ & 12.76 & 12.14 & 11.94 & 11.32 & 11.45 & 11.76 \\
\hline$l_{5}$ & 19.49 & 18.73 & 17.98 & 18.25 & 16.73 & 16.61 \\
\hline$l_{6}$ & 7.24 & 6.98 & 6.88 & 7.02 & 6.79 & 6.54 \\
\hline$l_{7}$ & 13.29 & 13.01 & 13.35 & 13.14 & 12.91 & 12.52 \\
\hline$l_{8}$ & 9.32 & 9.02 & 9.13 & 9.11 & 8.92 & 8.78 \\
\hline$l_{9}$ & 13.35 & 13.21 & 13.29 & 13.17 & 12.83 & 12.58 \\
\hline$w$ & 22.61 & 21.56 & 20.39 & 19.51 & 18.73 & 18.15 \\
\hline$w_{1}$ & 3.37 & 2.98 & 3.04 & 3.11 & 2.88 & 3.09 \\
\hline$w_{2}$ & 12.25 & 11.85 & 11.53 & 11.67 & 12.46 & 12.28 \\
\hline$w_{3}$ & 3.35 & 3.25 & 3.21 & 2.95 & 2.78 & 2.91 \\
\hline$w_{4}$ & 1.32 & 1.25 & 1.03 & 1.01 & 0.98 & 0.82 \\
\hline$w_{5}$ & 3.37 & 3.24 & 3.22 & 3.02 & 2.81 & 2.92 \\
\hline$w_{6}$ & 6.48 & 6.07 & 5.31 & 5.55 & 5.58 & 4.84 \\
\hline$w_{7}$ & 3.38 & 3.27 & 3.26 & 3.01 & 2.83 & 2.91 \\
\hline$w_{8}$ & 1.33 & 1.24 & 1.09 & 0.96 & 0.97 & 0.83 \\
\hline$w_{9}$ & 3.38 & 3.24 & 3.27 & 3.01 & 2.78 & 2.92 \\
\hline
\end{tabular}

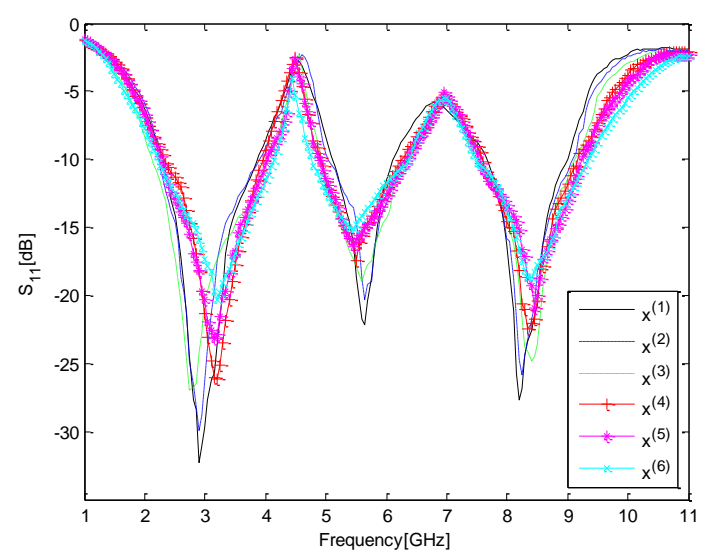

Fig. 5. Reflection responses at the selected Pareto-optimal designs

In order to verify the feasibility and validity of $\boldsymbol{R}_{\mathrm{s}}$, the results of $F_{1}$ for those selected Pareto-optimal designs, which are calculated by different approaches, are summarized in 
Table V. The predictive results 1 and predictive results 2 are given by $\boldsymbol{R}_{\mathrm{s}}$ without and with a dynamically updating surrogate model strategy, respectively. The error rate 1 and error rate 2 correspond to the two kinds of predictive results mentioned above in comparison with HFSS simulation results. Table $\mathrm{V}$ indicates that compared to predictive results 1, predictive results 2 are closer to the simulation results with an acceptable error rate (i.e., the average error rate is reduced from $8.56 \%$ to $5.29 \%$ ). It can be concluded that the proposed dynamically updating kriging model is an excellent substitute for time-consuming EM simulation process.

Table V Comparison of fitness values $F_{1}$ of selected Pareto-optimal designs obtained by $\boldsymbol{R}_{\mathrm{s}}$ and HFSS

\begin{tabular}{ccccccc}
\hline Design & $\boldsymbol{x}^{(1)}$ & $\boldsymbol{x}^{(2)}$ & $\boldsymbol{x}^{(3)}$ & $\boldsymbol{x}^{(4)}$ & $\boldsymbol{x}^{(5)}$ & $\boldsymbol{x}^{(6)}$ \\
\hline Simulation results & -16.87 & -15.96 & -15.12 & -14.54 & -13.93 & -13.24 \\
Predictive results 1 & -18.65 & -17.28 & -16.11 & -15.40 & -15.12 & -14.77 \\
Predictive results 2 & -18.16 & -16.76 & -15.65 & -14.02 & -13.28 & -12.29 \\
Error rate 1 & $10.55 \%$ & $8.27 \%$ & $6.55 \%$ & $5.91 \%$ & $8.54 \%$ & $11.56 \%$ \\
Error rate 2 & $7.65 \%$ & $5.02 \%$ & $3.51 \%$ & $3.71 \%$ & $4.67 \%$ & $7.18 \%$ \\
\hline \hline
\end{tabular}

In order to illustrate the evolution speed and diversity in design space, Fig. 6 presents the comparison of convergence curves among four algorithms (i.e., our improved MOEA/D, original MOEA/D [9], M-MOEA/D [23], and NSGA-II [17]). As for algorithm parameters, the population size $N$ is set to be 200 and the maximum number of iteration is set to be 150 . In all algorithms, the initial population is generated randomly and the same kriging-based surrogate strategy is used. It is observed that the improved MOEA/D, M-MOEA/D, and original MOEA/D show better convergence than NSGA-II because they acquire desired designs at a much faster speed. Moreover, the improved MOEA/D performs better in term of diversity than original MOEA/D and M-MOEA/D because it provides more desired designs at an acceptable iteration number. 


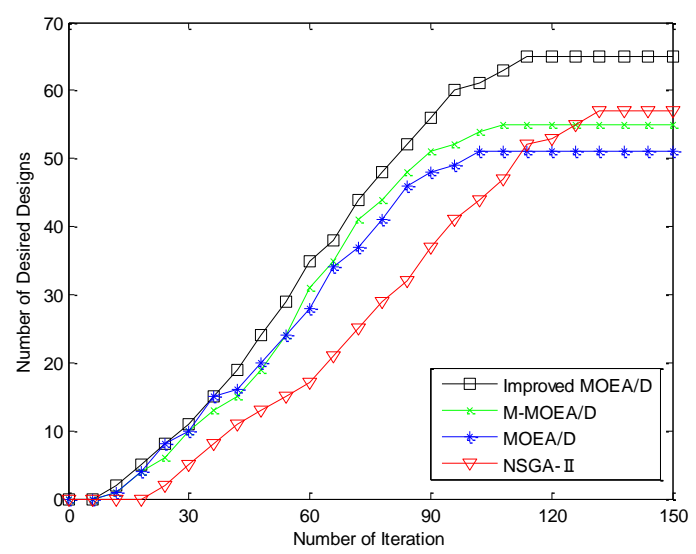

Fig. 6. Comparison of convergence curves using four different algorithms

Also, the proposed method is compared to other competitive techniques in terms of the CPU-time. EM simulation takes about 45 seconds each time based on simulation running environment which is equipped with 64-bit operating systems, 8GB RAM and $3.60 \mathrm{GHz}$ i7 processor. This work represents only $4.35 \%$ of the cost of the conventional EM simulation design (scheme 1), and direct multi-objective optimization using original MOEA/D without kriging interpolation (scheme 2) and with kriging interpolation (scheme 3) are about $19.03 \%$ and $6.42 \%$ of the cost of scheme 1 , respectively. The detailed cost breakdown is provided in Table VI. It is observed that the overall CPU-time required by our method is considerably lower than all benchmark techniques. Thus, the proposed method allows obtaining diverse desired designs without involving considerable computational resources.

TABLE VI Comparison of computational cost among different antenna optimization schemes

\begin{tabular}{cccc}
\hline \hline Optimization & Number of EM \\
approach & simulations & Total & Relative \\
\cline { 3 - 4 } & 14000 & 105 & $100 \%$ \\
Scheme 1 & 2500 & 19.98 & $19.03 \%$ \\
Scheme 2 & 750 & 6.74 & $6.42 \%$ \\
Scheme 3 & 520 & 4.57 & $4.35 \%$ \\
This work & & & \\
\hline \hline
\end{tabular}

\section{CONCLUSION}

In this paper, we propose an efficient and low-cost approach for fast multi-objective 
optimization of multi-parameter antenna structures. Firstly, an improved MOEA/D, which is a trade-off between population diversity and search speed, is proposed by designing diversity detection operation and mixed population update operation. The effectiveness of the improved MOEA/D has been demonstrated using several test functions. Further, a hybrid optimization strategy integrating a dynamically updatable surrogate-assisted model into the improved MOEA/D is proposed to greatly reduce computational cost of antenna optimization and maintain diversity simultaneously. Our approach is also applied to the design of a miniaturized multiband antenna, showing better diversity and significant savings of overall optimization cost compared with the previously reported design methods.

\section{ACKNOWLEDGMENTS}

This work was supported in part by the National Science Foundation of China under Grant No.61201086, in part by the China Scholarship Council under Grant No.201506375060, in part by the Planned Science and Technology Project of Guangdong Province under Grant 2013B090500007, and in part by the Dongguan Project on the Integration of Industry, Education and Research under Grant 2014509102205.

\section{REFERENCES}

[1] S. Koziel, S. Ogurtsov. Multi-objective design of antennas using variable-fidelity simulations and surrogate models. IEEE Trans. Antennas Prop., vol. 61, no. 12, pp. 5931-5939, 2013.

[2] S. Koziel, A. Bekasiwewicz. Fast multi-objective surrogate-assisted design of multi-parameter antenna structures through rotational design space reduction. IET Microwaves, Antennas \& Propagation, vol. 10, no. 6, pp. 624-630, 2016.

[3] R. Chirikov, P. Rocca, L. Manica, S. Santarelli, R. J. Mailloux, A. Massa. Innovative GA-based strategy for polyomino tiling in phased array design. European Conf. on Antenna \& Propag., pp. 2216-2219, 2013.

[4] M.A. Panduro, D.H. Covarrubias, C.A. Brizuela, F.R. Marante. A multi-objective approach in the linear antenna array design. AEU-International Journal of Electronics and Communications, vol. 59, no. 4, pp. 205-212, 2005. 
[5] Z. Lin, M. Yao, X. Shen, Sidelobe reduction of the low profile multi-subarray antenna by genetic algorithm, AEU-International Journal of Electronics and Communications, vol. 66, no. 2, pp. 133-139, 2012.

[6] Y. L. Li, W. Shao, L. You, B. Z. Wang. An Improved PSO Algorithm and Its Application to UWB Antenna Design. IEEE Antennas \& Wireless Propag. Lett., vol. 12, no. 3, pp.1236-1239, 2013.

[7] S. Chamaani, S. A. Mirtaheri, Planar UWB monopole antenna optimization to enhance time-domain characteristics using PSO, AEU-International Journal of Electronics and Communications, vol. 64, no. 4, pp. 351-359, 2010.

[8] Y. Kim, E. K. Walton. Automobile conformal antenna design using non-dominated sorting genetic algorithm (NSGA). IEE Proc.-Microw. Antennas Propag., vol. 153, no. 6, pp. 579-582, 2006.

[9] Q. Zhang, H. Li. MOED/A: A Multiobjective Evolutionary Algorithm Based on Decomposition. IEEE Trans. Evol. Comput., vol. 11, no. 6, pp. 712-731, 2007.

[10] X. Li, M. Yin. Design of multiobjective reconfigurable antenna array with discrete phase shifters using multiobjective differential evolution based on decomposition. International Journal of RF and Microwave Computer-Aided Engineering. vol. 22, no. 6, pp. 675-681, 2012.

[11] Y.K. Chen, S.W. Yang, Z.P. Nie. Improving conflicting specifications of time-modulated antenna arrays by using a multiobjective evolutionary algorithm. International Journal of Numerical Modelling-Electronic Networks Devices and Fields. vol. 25, no. 3, pp. 205-215, 2012.

[12] S. Koziel, A. Bekasiwewicz, W. Zieniutycz. Expedited EM-Driven Multiobjective Antenna Design in Highly Dimensional Parameter Spaces. IEEE Antennas \& Wireless Propag. Lett., vol. 13, pp. 631-634, 2014.

[13] S. Koziel, A. Bekasiewicz, I. Couckuyt, T. Dhaene. Efficient multi-objective simulation-driven antenna design using co-kriging. IEEE Trans. Antennas Prop., vol. 62, no. 11, pp. 5900-5905, 2014.

[14] A. Bekasiewicz, S. Koziel, W. Zieniutycz, and L. Leifsson. Expedited simulation-driven multi-objective design optimization of quasi-isotropic dielectric resonator antenna, in S. Koziel, L. Leifsson, X.S. Yang (Eds.) Simulation-driven modeling and optimization, Springer, pp. 207-232, 2016.

[15] S. Koziel, A. Bekasiwewicz. Scalability of surrogate-assisted multi-objective optimization of antenna structures exploiting variable-fidelity EM-simulation models. Engineering Optimization, vol. 48, no. 10, pp. 1778-1792, 2016.

[16] K. Deb. Multi-objective optimization using evolutionary algorithms. Wiley, New York, USA, 2001.

[17] K. Deb, A. Pratap, S. Agarwal, T. Meyarivan. A fast and elitist multi-objective genetic algorithm: NSGA-II. IEEE Trans. Evol. Comput., vol. 6, no. 2, pp. 182-197, 2002.

[18] N.V. Queipo, R.T. Haftka, W. Shyy, T. Goel, R. Vaidynathan, and P.K. Tucker. Surrogate-based analysis and optimization. Prog. Aerosp. Sci., vol. 41, no. 1, pp.1-28, 2005.

[19] M. Stein. Large sample properties of simulations using Latin hypercube sampling. Technometrics, vol. 29, no. 2, pp. 143-151, 1987.

[20] A. B. Owen. A central limit theorem for Latin hypercube sampling. Journal of the Royal Statistical Society:B, vol. 54, no. 2, pp. 541-551, 1992.

[21] E. Zitzler, K. Deb, L. Thiele. Comparison of multiobjective evolutionary algorithms:Empirical results. Evol. Comput., vol. 8, no. 2, pp. 173-195, 2000.

[22] K. Deb, L. Thiele, M. Laumanns, E. Zitzler. Scalable multiobjective optimization test problem. Proc. Congr. Evol. Comput, pp. 825-830, 2002.

[23] D. Ding, G. Wang. Modified Multiobjective Evolutionary Algorithm Based on Decomposition for Antenna Design. IEEE Trans. Antennas Propag., vol. 61, no. 10, pp. 5301-5307, 2013.

[24] E. Zitzler, L. Thiele, M. Laumanns, C. M. Fonseca, V. G. Fonseca. Performance assessment of 
multiobjective optimizers: An analysis and review. IEEE Trans. Evol. Comput, vol. 7, no. 2, pp. 117-132, 2003.

[25] J. Dong, X. Yu, G. Hu. Design of a compact quad-band slot antenna for integrated mobile devices. International Journal of Antennas and Propagation, vol. 2016, 2016. 